\title{
Modern Machine Learning Techniques and Their Applications to Medical Diagnostics
}

\author{
Alexander Gammerman \\ Computer Learning Research Centre, \\ Royal Holloway, University of London, UK \\ A.Gammerman@cs.rhul.ac.uk
}

\begin{abstract}
The talk presents several machine learning techniques and their applications to clinical decision-making. In many problems of computer-aided medical diagnosis and treatment a program must be capable of learning from previously accumulated past patients data records, and extrapolating to make diagnosis for new patient by considering their symptoms. Many machine learning and statisitical techniques have been developed to help in clinical decision making. Among them decision trees, the Bayesian techniques, dicriminant analysis, neural networks and many others. These techniques usually deal with conventional, small-scale, lowdimensional problems, and the application of these techniques to modern high-dimensional data sets with many thousand attributes (symptoms) usually leads to serious computational problems. Several new techniques such as Support Vector Machine (SVM) have been developed to tackle the problem of dimensionality by transferring the problem into highdimensional space, and solving it in that space. They based on so-called kernal methods and can very often solve some high-dimensional problems. These techniques perform very well with good accuracy. However, a typical drawback of techniques such as the SVM is that they usually do not provide any useful measure of confidence of new, unclassified examples (new pattients). Recently a new set of techniques, called Conformal Predictors, have been developed that allows to make predictions with valid measures of confidence. The approach is based on approximations to the universal measures of confidence given by the algorithmic theory of randomness and allows us to compute diagnostic classes and estimate confidence of the diagnostics for high-dimensional data. The talk will present Conformal Predictors and their applications in medicine.
\end{abstract}

